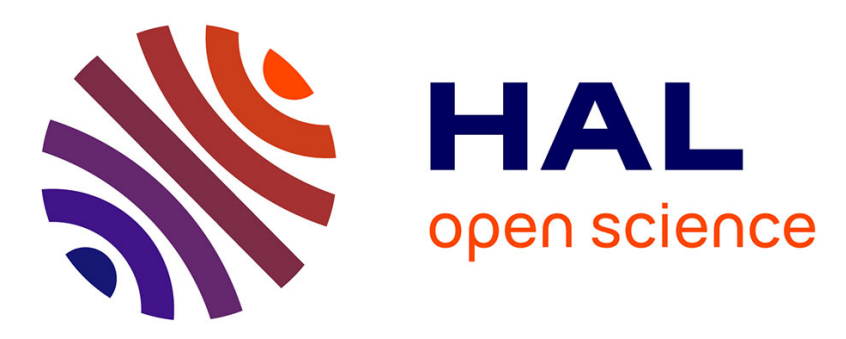

\title{
Joint Law of an Ornstein-Uhlenbeck Process and its Supremum
}

Christophette Blanchet-Scalliet, Diana Dorobantu, Laura Gay

\section{To cite this version:}

Christophette Blanchet-Scalliet, Diana Dorobantu, Laura Gay. Joint Law of an OrnsteinUhlenbeck Process and its Supremum. Journal of Applied Probability, 2020, 57 (2), pp.541-558. 10.1017/jpr.2020.22 . hal-01935756

\section{HAL Id: hal-01935756 https://hal.science/hal-01935756}

Submitted on 27 Nov 2018

HAL is a multi-disciplinary open access archive for the deposit and dissemination of scientific research documents, whether they are published or not. The documents may come from teaching and research institutions in France or abroad, or from public or private research centers.
L'archive ouverte pluridisciplinaire HAL, est destinée au dépôt et à la diffusion de documents scientifiques de niveau recherche, publiés ou non, émanant des établissements d'enseignement et de recherche français ou étrangers, des laboratoires publics ou privés. 


\title{
Joint Law of an Ornstein-Uhlenbeck Process and its Supremum
}

\author{
Christophette Blanchet-Scalliet* $\quad$ Diana Dorobantu ${ }^{\dagger} \quad$ Laura Gay $^{\ddagger}$
}

\begin{abstract}
Let $\left(X_{t}\right)_{t \geqslant 0}$ be an Ornstein-Uhlenbeck process driven by a Brownian motion. We propose an expression for the joint density / distribution function $\mathbb{P}\left(X_{t} \in \mathrm{d} x, \sup _{0 \leqslant u \leqslant t} X_{u} \leqslant m\right)$ where $m, x \in \mathbb{R}, x \leqslant m$. This law is expressed as an expansion involving parabolic cylinder functions. We obtain this law faster than with a Monte Carol's method. Numerical applications illustrate the interest of this result.
\end{abstract}

Keywords. Ornstein-Uhlenbeck process, joint law, supremum, endpoint, Fokker-Planck equation.

\section{Introduction}

The main purpose of this paper is to give an expression for the joint law of the endpoint and supremum of an Ornstein-Uhlenbeck process. The problem of finding such a joint law has attracted research since the late nineteenth century (see [Som94]). For a Brownian motion, the result is well known (see for example [KS91]). More recently, the authors of [EH14] give a method which allows to find this joint density / distribution function for the minimum and endpoints for a $n$-dimensional drifted Brownian motion. The explicit solution in the bidimensional case $(n=2)$ is given in [HKR98]. For $n>2$, the method in [EH14] works in only some particular cases. [CPN18] and [Ngo16] study the case of a Lévy process, sum of a Brownian motion with drift and a compound Poisson process. [CP17] are interested in the joint law of a $d$-dimensional diffusion process ans the running supremum of the first component. They find a PDE satisfied by the joint density (but an OrnsteinUhlenbeck process does not verify their assumptions). [DK06] study the general case of a Lévy process.

Let $\left(X_{t}\right)_{t \geqslant 0}$ be an Ornstein-Uhlenbeck process driven by a Brownian motion. Many results on the first passage time of an Ornstein-Uhlenbeck process have been obtained. In [APP05], the density function of the first hitting time to a fixed level by an Ornstein-Uhlenbeck process is given. Since hitting time and suprema are related, the cumulative distribution function of the supremum is obtained. Regarding the pair (process, its running maximum), [BS96] gives $\mathbb{P}\left(X_{\tau} \in \mathrm{d} x, \sup _{0 \leqslant u \leqslant \tau} X_{u} \geqslant m\right)$ where $\tau$ is a $\operatorname{Exp}(\lambda)$ r.v. independent of $X$. He also gives $\mathbb{P}\left(X_{t} \in \mathrm{d} x, \sup _{0 \leqslant u \leqslant t} X_{u} \leqslant 0\right), x \leqslant 0$. In this paper, we look for a more general case and we study the joint density / distribution function for an Ornstein-Uhlenbeck process driven by a Brownian motion, i.e. $\mathbb{P}\left(X_{t} \in \mathrm{d} x, \sup _{0 \leqslant u \leqslant t} X_{u} \leqslant m\right)$ where $m, x \in \mathbb{R}, x \leqslant m$. This law is solution of a Fokker-Planck equation. To solve this equation, a spectrum method is used and makes Weber's parabolic cylinder functions appear (directly linked with the Hermite functions). Even if the joint law is an infinite expansion of special functions, it is easy to evaluate numerically and it is faster than the Monte Carlo's method. We then check that our result is consistent with those in [BS96] and [APP05].

The paper is organized as follows. In the next section, some results including the main theorem with the expression of the joint density / distribution are presented. Section 3 is devoted to the proof of this theorem. Finally, Section 4 is concerned with the law of the supremum and its joint density with the process.

\section{Results}

\subsection{Context and notations}

Let $\left(B_{t}\right)_{t \geqslant 0}$ be a standard Brownian motion defined on a probability space $(\Omega, \mathcal{F}, \mathbb{P})$. We consider an OrnsteinUhlenbeck (OU in the following) process $\left(X_{t}\right)_{t \geqslant 0}$ solution of the following SDE :

$$
\mathrm{d} X_{t}=-k X_{t} \mathrm{~d} t+\mathrm{d} B_{t}, \quad X_{0} \sim \mu
$$

\footnotetext{
*University of Lyon, CNRS UMR 5208, Ecole Centrale de Lyon, Institut Camille Jordan, France, christophette.blanchet@eclyon.fr

${ }^{\dagger}$ University of Lyon, University Lyon 1, ISFA, LSAF (EA 2429) France, diana.dorobantu@univ-lyon1.fr

${ }^{\ddagger}$ University of Lyon, CNRS UMR 5208, Ecole Centrale de Lyon, Institut Camille Jordan, France, laura.gay@ec-lyon.fr
} 
where $k \in \mathbb{R}_{+}^{*}$ and $\mu$ is a probability measure. Suppose that $X_{0}$ and $\left(B_{t}\right)_{t \geqslant 0}$ are independent.

Let $\mathbb{P}_{x_{0}}$ be the law of the process $\left(X_{t}\right)_{t \geqslant 0}$ when $X_{0}=x_{0}$.

\subsection{Main theorem}

Our aim is to compute the joint law of the process and its supremum. However, as it is not easy to compute the joint density, we are first interested in the joint density / distribution

$$
\mathbb{P}\left(X_{t} \in \mathrm{d} x, \sup _{0 \leqslant u \leqslant t} X_{u} \leqslant m\right) \text { where } t \in \mathbb{R}_{+}^{*},(m, x) \in \mathbb{R}^{2}, x \leqslant m .
$$

The following theorem gives an expression for the joint density / distribution.

Theorem 2.1. We assume that $\mu$ is a probability measure on $]-\infty, m], m \in \mathbb{R}$ and set $p_{0}$ such that $\mu(\mathrm{d} x)=p_{0}(x) \mathrm{d} x$.

(i) For all $x \leqslant m$ and $t \in \mathbb{R}_{+}$, the probability $\mathbb{P}\left(X_{t} \in \mathrm{d} x, \sup _{0 \leqslant u \leqslant t} X_{u} \leqslant m\right)$ admits a density $p$ :

$$
\mathbb{P}\left(X_{t} \in \mathrm{d} x, \sup _{0 \leqslant u \leqslant t} X_{u} \leqslant m\right)=p(m, x, t) \mathrm{d} x .
$$

(ii) The density $p$ solves the following Fokker-Planck equation

$$
\forall m \in \mathbb{R},\left\{\begin{array}{ccc}
\partial_{t} p(m, x, t) & =k \partial_{x}(x p(m, x, t))+\frac{1}{2} \partial_{x^{2}}^{2} p(m, x, t), & (x, t) \in]-\infty, m] \times \mathbb{R}_{+}^{*} \\
p(m, m, t) & =0, & t \in \mathbb{R}_{+}^{*} \\
p(m, x, 0) & =p_{0}(x), & x \in]-\infty, m] .
\end{array}\right.
$$

(iii) For $(m, x) \in\left\{(z, y) \in \mathbb{R}^{2} \mid z \geqslant y\right\}, t \in \mathbb{R}_{+}^{*}$, the density $p$ can be expressed as :

$$
p(m, x, t)=-\sqrt{2 k} \mathrm{e}^{-\frac{k x^{2}}{2}} \sum_{n=1}^{\infty} \mathrm{e}^{-k \nu_{n, m} t} \frac{D_{\nu_{n, m}}(-x \sqrt{2 k}) \int_{-\infty}^{m} \mathrm{e}^{\frac{k z^{2}}{2}} p_{0}(z) D_{\nu_{n, m}}(-z \sqrt{2 k}) \mathrm{d} z}{\nu_{n, m} D_{\nu_{n, m}-1}(-m \sqrt{2 k}) \partial_{\nu} D_{\nu_{n, m}}(-m \sqrt{2 k})},
$$

where $D_{\nu}$ is the parabolic cylinder function and $\left(\nu_{j, m}\right)_{j \geqslant 1}$ is the ordered sequence of positive zeros of $\nu \mapsto D_{\nu}(-m \sqrt{2 k})$ (details are given in Appendix).

The proof of the theorem is postponed to Section 3. We start to study some particular cases and consequences.

Remark 2.2. For $(m, x) \in\left\{(z, y) \in \mathbb{R}^{2} \mid z \geqslant y\right\}, t \in \mathbb{R}_{+}^{*}$, when $X_{0}=x_{0} \leqslant m$,

$$
p(m, x, t)=-\sqrt{2 k} \mathrm{e}^{\frac{k\left(x_{0}^{2}-x^{2}\right)}{2}} \sum_{n=1}^{\infty} \mathrm{e}^{-k \nu_{n, m} t} \frac{D_{\nu_{n, m}}\left(-x_{0} \sqrt{2 k}\right) D_{\nu_{n, m}}(-x \sqrt{2 k})}{\nu_{n, m} D_{\nu_{n, m}-1}(-m \sqrt{2 k}) \partial_{\nu} D_{\nu_{n, m}}(-m \sqrt{2 k})} .
$$

The expression of the joint density / distribution can be easily extended to an OU process depending on 3 parameters.

Corollary 2.3. Suppose the OU process $\left(X_{t}\right)_{t \geqslant 0}$ solution of the following SDE :

$$
\mathrm{d} X_{t}=\left(\phi-k X_{t}\right) \mathrm{d} t+\sqrt{\beta} \mathrm{d} B_{t}, \quad X_{0} \sim \mu
$$

where $\beta, k \in \mathbb{R}_{+}^{*}$ and $\phi \in \mathbb{R}$.

For $(m, x) \in\left\{(z, y) \in \mathbb{R}^{2} \mid z \geqslant y\right\}, t \in \mathbb{R}_{+}^{*}$, the density $p$ can be expressed as :

$$
\begin{aligned}
p(m, x, t)=-\sqrt{\frac{2 k}{\beta}} \mathrm{e}^{-\frac{k\left(x-\frac{\phi}{k}\right)^{2}}{2 \beta}} \sum_{n=1}^{\infty} & \left(\int_{-\infty}^{m-\frac{\phi}{k}} \mathrm{e}^{\frac{k z^{2}}{2 \beta}} p_{0}(z) D_{\nu_{n, m-\frac{\phi}{k}}}\left(-z \sqrt{\frac{2 k}{\beta}}\right) \mathrm{d} z\right) \mathrm{e}^{-k \nu_{n, m-\frac{\phi}{k}} t} \\
& \times \frac{D_{\nu_{n, m-\frac{\phi}{k}}}\left(-\left(x-\frac{\phi}{k}\right) \sqrt{2 \frac{k}{\beta}}\right)}{\nu_{n, m-\frac{\phi}{k}} D_{\nu_{n, m-\frac{\phi}{k}}}\left(-\left(m-\frac{\phi}{k}\right) \sqrt{\frac{2 k}{\beta}}\right) \partial_{\nu} D_{\nu_{n, m-\frac{\phi}{k}}}\left(-\left(m-\frac{\phi}{k}\right) \sqrt{\frac{2 k}{\beta}}\right.} .
\end{aligned}
$$

where $D_{\nu}$ is the parabolic cylinder function and $\left(\nu_{j, m-\frac{\phi}{k}}\right)_{j \geqslant 1}$ is the ordered sequence of positive zeros of $\nu \mapsto D_{\nu}\left(-\left(m-\frac{\phi}{k}\right) \sqrt{\frac{2 k}{\beta}}\right)$. 
Proof : Let set $U_{t}=X_{\frac{t}{\beta}}-\frac{\phi}{k}$ and $W_{t}=\sqrt{\beta} B_{\frac{t}{\beta}}$ (which is thus a standard Brownian motion). Then the dynamic of $\left(U_{t}\right)_{t \geqslant 0}$ is

$$
\mathrm{d} U_{t}=-\frac{k}{\beta} U_{t} \mathrm{~d} t+\mathrm{d} W_{t}, \quad U_{0}=X_{0}-\frac{\phi}{k} \in \mathbb{R} .
$$

We conclude with Theorem 2.1.

\subsection{Numerical results}

Even though Formula (2) is an infinite sum of special functions, it is easy to evaluate numerically.

The function $x \mapsto p(m, x, t)$ is evaluated with expression (2) (or (3)) and is compared with a Monte Carlo's method using $5 \times 10^{5}$ simulations. Figures 1 and 2 plot $\mathbb{P}\left(X_{t} \in \mathrm{d} x, \sup _{0 \leqslant u \leqslant t} X_{u} \leqslant m\right)$ for different parameters and initial laws with $t=1$.

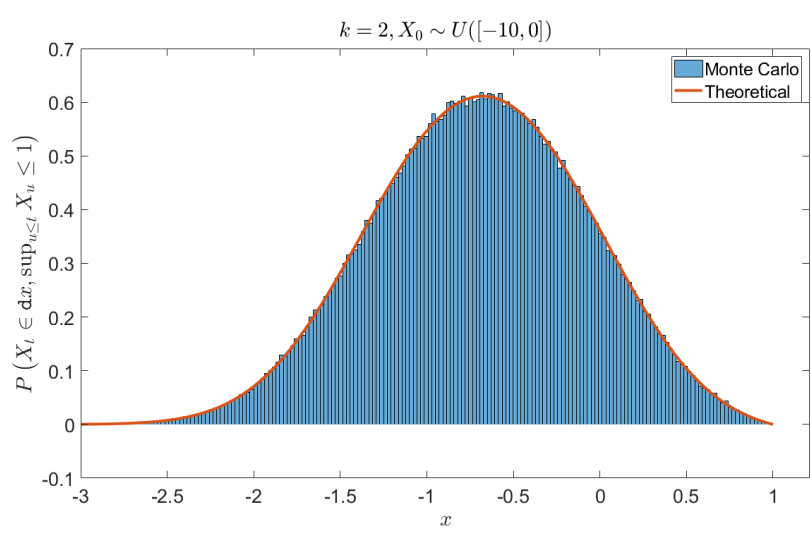

Figure 1: Comparison of expression (2) and the Monte Carlo's method when $m=1$, $X_{0} \sim \mathcal{U}([-10,0])$ and $k=2$.

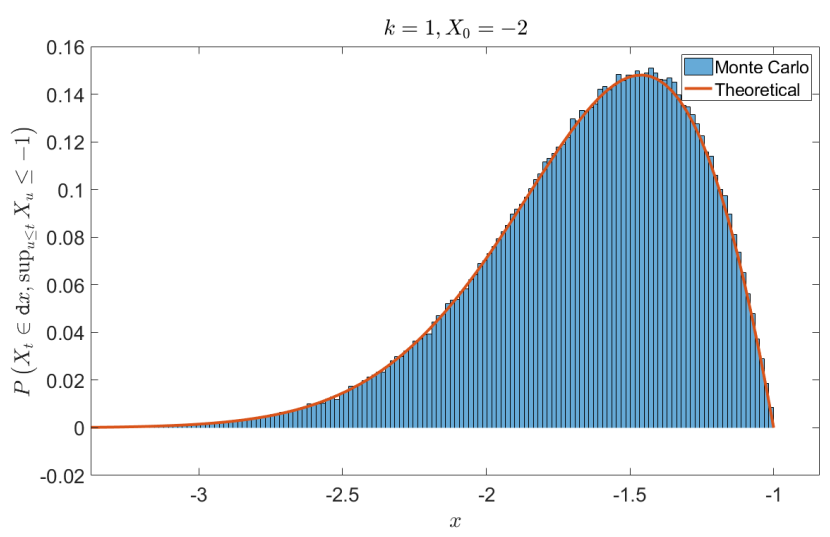

Figure 2: Comparison of expression (3) and the Monte Carlo's method when $m=-1, X_{0}=-2$ and $k=1$.

Last but not least, our formula is faster than the Monte Carlo's method. Indeed, with a simple core, obtaining the whole function $x \mapsto p(m, x, t)$ lasts around $23 \mathrm{sec}$ whereas a Monte Carlo's method with $10^{5}$ simulations lasts around $18 \mathrm{sec}, 2.10^{5}$ simulations $34 \mathrm{sec}$ and $10^{6}$ simulations $176 \mathrm{sec}$.

\subsection{Particular case $m=0$}

When $m=0$, the expression of the joint density / distribution can be simplified. Indeed, the zeros $\left(\nu_{n, 0}\right)_{n \geqslant 0}$ of $\nu \mapsto D_{\nu}(0)$ are the positive odd integers (see Appendix for more details). In this particular case, Formula (3) can be simplified.

Proposition 2.4. We assume $X_{0}=x_{0} \leqslant 0$. For all $x \leqslant 0$ and $t \in \mathbb{R}_{+}^{*}$,

$$
\mathbb{P}_{x_{0}}\left(X_{t} \in \mathrm{d} x, \sup _{0 \leqslant s \leqslant t} X_{s} \leqslant 0\right)=\sqrt{\frac{k}{\pi\left(1-\mathrm{e}^{-2 k t}\right)}}\left[\mathrm{e}^{-k \frac{\left(x-x_{0} \mathrm{e}^{-k t}\right)^{2}}{1-\mathrm{e}^{-2 k t}}}-\mathrm{e}^{-k \frac{\left(x+x_{0} \mathrm{e}^{-k t}\right)^{2}}{1-\mathrm{e}^{-2 k t}}}\right] \mathrm{d} x .
$$

Proof : Without loss of generality, $k=1$ is taken in the proof. We use the results given in Appendix. With (29) and Proposition 6.2, we have :

$$
p(0, x, t)=\frac{2 \mathrm{e}^{\frac{x_{0}^{2}-x^{2}}{2}}}{\sqrt{\pi}} \sum_{n=0}^{\infty} \mathrm{e}^{-(2 n+1) t} \frac{D_{2 n+1}\left(-x_{0} \sqrt{2}\right) D_{2 n+1}(-x \sqrt{2})}{(2 n+1) !} .
$$

We use (28) and then, with a Fubini theorem (everything is clearly integrable),

$$
p(0, x, t)=-\frac{2 \mathrm{e}^{x_{0}^{2}}}{\pi \sqrt{\pi}} \sum_{n=0}^{\infty} \frac{2^{2 n+1} \mathrm{e}^{-(2 n+1) t}}{(2 n+1) !} \int_{0}^{\infty} \int_{0}^{\infty}(u v)^{2 n+1} \mathrm{e}^{-\left(u^{2}+v^{2}\right)}\left(\mathrm{e}^{2 \mathrm{i} v x_{0}}-\mathrm{e}^{-2 \mathrm{i} v x_{0}}\right)\left(\mathrm{e}^{2 \mathrm{i} u x}-\mathrm{e}^{-2 \mathrm{i} u x}\right) \mathrm{d} u \mathrm{~d} v .
$$


We can invert integral and sum :

$$
\begin{aligned}
p(0, x, t) & =-\frac{2 \mathrm{e}^{x_{0}^{2}}}{\pi \sqrt{\pi}} \int_{0}^{\infty} \int_{0}^{\infty} \mathrm{e}^{-\left(u^{2}+v^{2}\right)}\left(\mathrm{e}^{2 \mathrm{i} v x_{0}}-\mathrm{e}^{-2 \mathrm{i} v x_{0}}\right)\left(\mathrm{e}^{2 \mathrm{i} u x}-\mathrm{e}^{-2 \mathrm{i} u x}\right) \operatorname{sh}\left(2 u v \mathrm{e}^{-t}\right) \mathrm{d} u \mathrm{~d} v \\
& =-\frac{\mathrm{e}^{x_{0}^{2}}}{\pi \sqrt{\pi}}\left[\int_{-\infty}^{\infty} \mathrm{e}^{-v^{2}+2 \mathrm{i} v x_{0}} \int_{-\infty}^{\infty} \mathrm{e}^{-u^{2}+2 \mathrm{i} u x+2 u v \mathrm{e}^{-t}} \mathrm{~d} u \mathrm{~d} v-\int_{-\infty}^{\infty} \mathrm{e}^{-v^{2}+2 \mathrm{i} v x_{0}} \int_{-\infty}^{\infty} \mathrm{e}^{-u^{2}+2 \mathrm{i} u x-2 u v \mathrm{e}^{-t}} \mathrm{~d} u \mathrm{~d} v\right]
\end{aligned}
$$

Finally, formula (4.11.6) in [LS72] gives :

$$
\begin{aligned}
p(0, x, t) & =-\frac{\mathrm{e}^{x_{0}^{2}}}{\pi}\left[\int_{-\infty}^{\infty} \mathrm{e}^{-v^{2}+2 \mathrm{i} v x_{0}} \mathrm{e}^{\left(\mathrm{i} x+v \mathrm{e}^{-t}\right)^{2}} \mathrm{~d} v-\int_{-\infty}^{\infty} \mathrm{e}^{-v^{2}+2 \mathrm{i} v x_{0}} \mathrm{e}^{\left(\mathrm{i} x-v \mathrm{e}^{-t}\right)^{2}} \mathrm{~d} v\right] \\
& =-\frac{\mathrm{e}^{x_{0}^{2}-x^{2}}}{\sqrt{\pi\left(1-\mathrm{e}^{-2 t}\right)}}\left[\mathrm{e}^{-\frac{\left(x_{0}+x \mathrm{e}^{-t}\right)^{2}}{1-\mathrm{e}^{-2 t}}}-\mathrm{e}^{-\frac{\left(x_{0}-x \mathrm{e}^{-t}\right)^{2}}{1-\mathrm{e}^{-2 t}}}\right] \\
& =\frac{1}{\sqrt{\pi\left(1-\mathrm{e}^{-2 t}\right)}}\left[\mathrm{e}^{-\frac{\left(x-x_{0} \mathrm{e}^{-t}\right)^{2}}{1-\mathrm{e}^{-2 t}}}-\mathrm{e}^{-\frac{\left(x+x_{0} \mathrm{e}^{-t}\right)^{2}}{1-\mathrm{e}^{-2 t}}}\right] .
\end{aligned}
$$

Remark 2.5. This is formula (1.1.8) of Chapter 7 in [BS96]. Note that there is a typography in the book.

\section{Proof of the main theorem}

This section is devoted to the proof of Theorem 2.1. The proof is divided into two parts. First, we prove the existence of the density and the fact that it is solution of the Fokker Planck Equation. The proof of the theorem relies on the second part namely resolution of this Fokker Planck equation. Without loss of generality, we take here $k=1$.

\section{Existence of the density and the Fokker-Planck equation}

(i) The existence of the density is given by Lemma 2.1 in [DIRT13].

(ii) It is just Proposition 5.4.3.1 in [JYC09] with $h: t \mapsto m$.

\section{Resolution of the Fokker-Planck equation}

(iii) Now, we want to solve the Fokker-Planck equation. We use a spectrum method inspired by [BD08], [Gar04] and [RH89]. In that purpose, we introduce two operators.

Let us note $\left.\left.\left.\left.\mathcal{D}\left(\mathbf{L}_{\mathrm{FP}}\right):=\left\{f \in H^{1}(]-\infty, m\right]\right) \mid\left[x \mapsto \frac{\partial}{\partial x}(x f(x))+\frac{1}{2} f^{\prime \prime}(x)\right] \in L^{2}(]-\infty, m\right]\right), f(m)=0\right\}$.

The Fokker Planck operator $\mathbf{L}_{\mathrm{FP}}$ is defined by :

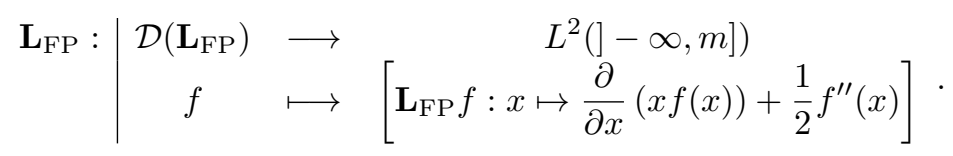

The goal is to find a basis $\left(\tilde{\varphi}_{n}\right)_{n}$ of eigenvectors of $\mathbf{L}_{\mathrm{FP}}$ in order to write

$$
p(m, x, t)=\sum_{n=1}^{\infty} c_{n}(t) \tilde{\varphi}_{n}(x) .
$$

The Fokker-Planck operator $\mathbf{L}_{\mathrm{FP}}$ is not hermitian. Then, to build this basis, we introduce the following operator $\mathbf{O}$, related to $\mathbf{L}_{\mathrm{FP}}$, defined by :

$$
\mathbf{O}: \mid \begin{array}{clc}
\mathcal{D}\left(\mathbf{L}_{\mathrm{FP}}\right) & \longrightarrow \\
f & \longmapsto & \left.\left.L^{2}(]-\infty, m\right]\right) \\
& \left.\mathbf{O} f: x \mapsto-\frac{1}{2} x^{2} f(x)+\frac{1}{2} f^{\prime \prime}(x)\right]
\end{array}
$$

As $\mathbf{O}$ is hermitian, we can use classical results on hermitian operators decomposition.

Moreover, we have a correspondence for the spectrum. The function $\phi$ is an eigenfunction of $\mathbf{O}$ with eigenvalue $\mu$ if and only if $x \mapsto \mathrm{e}^{-\frac{x^{2}}{2}} \phi$ is an eigenfunction of $\mathbf{L}_{\mathrm{FP}}$ with eigenvalue $\mu+\frac{1}{2}$. 
Hilbertian basis of $\left.\left.L^{2}(]-\infty, m\right]\right)$

We prove that any function of $\left.\left.L^{2}(]-\infty, m\right]\right)$ can be decomposed on an hilbertian basis of eigenvectors of $\mathbf{O}$. Since it is not easy to prove this directly with the operator $\mathbf{O}$, it is classic (see proof of Theorem VIII.20 in [Bré83] for instance) to introduce the operator $\mathbf{T}$ defined as :

$$
\mathbf{T}: \mid \begin{array}{ccc}
\left.\left.L^{2}(]-\infty, m\right]\right) & \longrightarrow & \left.\left.L^{2}(]-\infty, m\right]\right) \\
g & \longmapsto & f_{g},
\end{array}
$$

where $f_{g}$ is solution of

$$
\left\{\begin{array}{ccc}
\frac{1}{2} f_{g}^{\prime \prime}-\frac{1}{2} x^{2} f_{g} & = & g \\
f_{g}(m) & = & 0 \\
f_{g} & \in & \left.\left.H^{1}(]-\infty, m\right]\right) .
\end{array}\right.
$$

To prove that $\mathbf{T}$ is well defined, we need to introduce the following space $V$ and we give some properties on this space. Let us note

$$
\left.\left.\left.\left.\left.\left.V:=\left\{v \in L^{2}(]-\infty, m\right]\right) \mid v^{\prime} \in L^{2}(]-\infty, m\right]\right), v(m)=0, x \mapsto x v \in L^{2}(]-\infty, m\right]\right)\right\}
$$

endowed with the scalar product $\langle u, v\rangle_{V}=\left\langle u^{\prime}, v^{\prime}\right\rangle_{\left.\left.L^{2}(]-\infty, m\right]\right)}+\langle x u, x v\rangle_{\left.\left.L^{2}(]-\infty, m\right]\right)}$. The space $V$ is a weighted Sobolev space. With its scalar product, the space $V$ is then an Hilbert space on $\mathbb{R}$.

The following lemma generalizes in our context a classical inequality for Sobolev spaces.

Lemma 3.1. If $f \in V$, there exists a constant $C \in \mathbb{R}$ such that

$$
\|f\|_{\left.\left.L^{2}(]-\infty, m\right]\right)} \leqslant C\|f\|_{V}
$$

Proof : If $m \geqslant-1$, we have

$$
\begin{aligned}
\|f\|_{\left.\left.L^{2}(]-\infty, m\right]\right)}^{2} & \leqslant \int_{-\infty}^{-1} f^{2} x^{2} \times \underbrace{\frac{1}{x^{2}}}_{\leqslant 1} \mathrm{~d} x+\underbrace{\tilde{C}\left\|f^{\prime}\right\|_{L^{2}([-1, m])}^{2}}_{\text {Poincaré's inequality because } f(m)=0} \\
& \leqslant C\|f\|_{V}^{2} .
\end{aligned}
$$

If $m<-1$, it is easy to adapt the previous proof .

We prove that the operator $\mathbf{T}$ is well defined thanks to the Lax-Milgram theorem. For a fixed function $\left.\left.g \in L^{2}(]-\infty, m\right]\right)$, the weak formulation of $(4)$ is, for $\left.\left.\varphi \in H_{0}^{1}(]-\infty, m\right]\right)$,

$$
\left\{\begin{array}{cccc}
a(f, \varphi) & = & l(\varphi) \\
f(m) & = & 0 \\
f & \in & \left.\left.H^{1}(]-\infty, m\right]\right)
\end{array}\right.
$$

with $a(f, \varphi)=-\frac{1}{2}\left(\int_{-\infty}^{m} f^{\prime} \varphi^{\prime}+\int_{-\infty}^{m} x^{2} f \varphi\right)$ et $l(\varphi)=\int_{-\infty}^{m} g \varphi$.

We check the hypothesis of the Lax-Milgram's theorem. The application $a$ is clearly bilinear and coercive. It is continuous thanks to the Cauchy-Schwarz inequality. Finally, (5) gives that $l$ is a linear continuous form on $V$.

The Lax-Milgram's theorem allows to conclude that there exists an unique solution $f \in V$ to $a(f, \varphi)=l(\varphi)$ and then the operator $\mathbf{T}$ is well defined.

Proposition 3.2. The space $\left.\left.L^{2}(]-\infty, m\right]\right)$ admits an hilbertian basis of eigenvectors of $\boldsymbol{O}$ for the scalar product associated to the $L^{2}$-norm.

Proof : First, we prove that $\left.\left.L^{2}(]-\infty, m\right]\right)$ admits an hilbertian basis of eigenvectors of T. We prove that the operator $\mathbf{T}$ is self-adjoint and compact so that we have an hilbertian basis.

The operator $\mathbf{T}$ is self-adjoint. It is just an integration by parts. 
The operator $\mathbf{T}$ is compact. To prove that the operator $\mathbf{T}$ is linear, we use the uniqueness of the solution implied by Lax-Milgram's theorem. Then, $\mathbf{T}(g)=f_{g} \in V$ and with (5)

$$
\|\mathbf{T}(g)\|_{\left.\left.L^{2}(]-\infty, m\right]\right)}=\left\|f_{g}\right\|_{\left.\left.L^{2}(]-\infty, m\right]\right)} \leqslant C\left\|f_{g}\right\|_{V} .
$$

However, multiplying (4) by $f_{g}$ and integrating, we have

$$
a\left(f_{g}, f_{g}\right)=l\left(f_{g}\right) \Rightarrow \frac{1}{2}\left\|f_{g}\right\|_{V}^{2} \leqslant C\left\|f_{g}\right\|_{V} \Rightarrow\left\|f_{g}\right\|_{V} \leqslant 2 C .
$$

Then, $\mathbf{T}$ is continuous. The compacity of $\mathbf{T}$ follows from the compact injection of $V$ in $\left.\left.L^{2}(]-\infty, m\right]\right)$ (this can be proved using Corollary IV.26 in [Bré83]).

Conclusion $\mathbf{T}$ is a self-adjoint compact operator. Then, by Theorem VI.11. in [Bré83], as $\left.\left.L^{2}(]-\infty, m\right]\right)$ is a separable space, $\left.\left.L^{2}(]-\infty, m\right]\right)$ admits an hilbertian basis of eigenvectors of $\mathbf{T}$ for the scalar product of $\left.\left.L^{2}(]-\infty, m\right]\right)$.

However, the eigenvectors of $\mathbf{T}$ are the eigenvectors of $\mathbf{O}$ with inverse eigenvalues and we can conclude the proof.

\section{Eigenvectors of $\mathrm{O}$}

Let $\phi$ be an eigenvector of $\mathbf{O}$ with eigenvalue $\mu$. We have

$$
\frac{1}{2} \phi^{\prime \prime}(x)-\frac{1}{2} x^{2} \phi(x)=\mu \phi(x) .
$$

We then apply the variable change $z=x \sqrt{2}$ and $h: z \mapsto \phi\left(\frac{z}{\sqrt{2}}\right)=\phi(x)$. The equation for $h$ is

$$
h^{\prime \prime}(z)+\left(-\frac{1}{4} z^{2}-\mu\right) h(z)=0 .
$$

As indicated in Appendix (see Section 6.1), the solutions for this equation can be written as $h=\alpha h_{1}+\beta h_{2}$ where $h_{1}(z)=D_{-\mu-\frac{1}{2}}(z)$ and $h_{2}(z)=D_{-\mu-\frac{1}{2}}(-z)$ and $D$ is the parabolic cylinder function defined in (26). Unlike $h_{1}, h_{2}$ is integrable on $-\infty$.

Then, we take $\phi: x \mapsto D_{-\mu-\frac{1}{2}}(-x \sqrt{2})$.

As $\phi \in \mathcal{D}\left(\mathbf{L}_{\mathrm{FP}}\right)$, we must have $\phi(m)=0$, i.e.

$$
D_{-\mu-\frac{1}{2}}(-m \sqrt{2})=0
$$

\section{Eigenvalues of $\mathrm{O}$}

Thanks to the previous paragraph, we have $\operatorname{Sp}(\mathbf{O})=\left\{-\nu-\frac{1}{2} \mid D_{\nu}(-m \sqrt{2})=0\right\}$. We prove that the spectrum of $\mathbf{O}$ is included in $\left.]-\infty,-\frac{1}{2}\right]$.

We recall that

$$
\langle\mathbf{O} f, f\rangle_{\left.\left.L^{2}(]-\infty, m\right]\right)}=-\frac{1}{2}\left(\int_{-\infty}^{m} x^{2} f^{2}(x) \mathrm{d} x+\int_{-\infty}^{m} f^{\prime 2}(x) \mathrm{d} x\right) .
$$

Let us study $\operatorname{Sp}(\mathbf{O})$ for the different values of $m$.

- if $m \leqslant-1$ : We easily obtain $\langle\mathbf{O} f, f\rangle_{\left.\left.L^{2}(]-\infty, m\right]\right)}<-\frac{1}{2}\langle f, f\rangle$ and $\left.\operatorname{so} \operatorname{Sp}(\mathbf{O}) \subset\right]-\infty,-\frac{1}{2}[$. 
- if $-1<m \leqslant 0$ : We have

$$
\begin{aligned}
\int_{-\infty}^{m} f^{2} & =\int_{-\infty}^{-1} f^{2}+\int_{-1}^{m} f^{2} \\
& \leqslant \int_{-\infty}^{-1} f^{2} x^{2} \mathrm{~d} x+\int_{-1}^{m}\left(\int_{x}^{m} f^{\prime}(t) \mathrm{d} t\right)^{2} \mathrm{~d} x \\
& \stackrel{\text { C.S. }}{\leqslant} \int_{-\infty}^{-1} f^{2} x^{2} \mathrm{~d} x+\left\|f^{\prime}\right\|_{\left.\left.L^{2}(]-\infty, m\right]\right)}^{2} \int_{-1}^{m}(m-x)^{2} \mathrm{~d} x \\
& =\int_{-\infty}^{-1} f^{2} x^{2} \mathrm{~d} x+\left\|f^{\prime}\right\|_{\left.\left.L^{2}(]-\infty, m\right]\right)}^{2} \frac{(m+1)^{3}}{3}, \\
\langle f, f\rangle & <-2\langle\mathbf{O} f, f\rangle_{\left.\left.L^{2}(]-\infty, m\right]\right)} .
\end{aligned}
$$

Then, if $-1<m \leqslant 0,\langle\mathbf{O} f, f\rangle_{\left.\left.L^{2}(]-\infty, m\right]\right)}<-\frac{1}{2}\langle f, f\rangle$ and so $\left.\operatorname{Sp}(\mathbf{O}) \subset\right]-\infty,-\frac{1}{2}[$.

- if $m>0$ : Let $\mu$ be an eigenvalue of $\mathbf{O}$. Using a proof by contradiction, let us assume that $\mu \geqslant-\frac{1}{2}$. Then, by (7) and formula (19) in Appendix, as $H_{-\mu-\frac{1}{2}}(-m)=0$, we would have

$$
\sum_{i=0}^{+\infty} \frac{\Gamma\left(\frac{i+\mu+\frac{1}{2}}{2}\right)}{i !}(2 m)^{i}=0 .
$$

This is absurd because all the terms are non-negative. Then, we have necessarily $\mu<-\frac{1}{2}$ when $m>0$.

Finally, for all $m \in \mathbb{R}, \operatorname{Sp}(\mathbf{O}) \subset]-\infty,-\frac{1}{2}\left[\right.$ and hence $\left\{\nu \mid D_{\nu}(-m \sqrt{2})=0\right\} \subset \mathbb{R}_{+}^{*}$.

\section{Expression of $p$}

We have found the eigenvectors of $\mathbf{O}$ namely the functions $x \mapsto D_{\nu_{n, m}}(-x \sqrt{2})$ with $\left(\nu_{n, m}\right)_{n \in \mathbb{N}^{*}}$ the ordered positive roots of $\nu \mapsto D_{\nu}(-m \sqrt{2})$.

With a normalisation, we finally have an hilbertian basis $\left(\tilde{e}_{n}\right)_{n \in \mathbb{N}^{*}}$ of $\left.\left.L^{2}(]-\infty, m\right]\right)$ with the scalar product of $\left.\left.L^{2}(]-\infty, m\right]\right)$. It is defined by

$$
\tilde{e}_{n}: x \mapsto \frac{D_{\nu_{n, m}}(-x \sqrt{2})}{\sqrt{\int_{-\infty}^{m} D_{\nu_{n, m}}^{2}(-y \sqrt{2}) \mathrm{d} y}} .
$$

Now, it remains to go back to $\mathbf{L}_{\mathrm{FP}}$ to find the form of $p$.

Let us note $\tilde{\varphi}_{n}(x):=\mathrm{e}^{-\frac{x^{2}}{2}} \tilde{e}_{n}(x)$. We recall that $\tilde{\varphi}_{n}$ is an eigenfunction of $\mathbf{L}_{\mathrm{FP}}$ with eigenvalue $-\nu_{n, m}$. It is easy to prove that $\left(\tilde{\varphi}_{n}\right)_{n \in \mathbb{N}^{*}}$ is an hilbertian basis of $\left.\left.L^{2}(]-\infty, m\right], \mathrm{e}^{x^{2} / 2}\right)$ with the scalar product

$$
\langle f, g\rangle_{0}=\int_{-\infty}^{m} \mathrm{e}^{x^{2}} f(x) g(x) \mathrm{d} x .
$$

We look for a solution $p$ in $\left.\left.L^{2}(]-\infty, m\right], \mathrm{e}^{x^{2} / 2}\right)$. We can then decompose $x \mapsto p(m, x, t)$ on the basis of $\left(\tilde{\varphi}_{n}\right)_{n \in \mathbb{N}^{*}}$ :

$$
p(m, x, t)=\sum_{n=1}^{\infty} c_{n}(t) \tilde{\varphi}_{n}(x) .
$$

However,

$$
p(m, x, 0)=\sum_{n=1}^{\infty} c_{n}(0) \tilde{\varphi}_{n}(x)
$$


Then,

$$
\begin{aligned}
c_{n}(0) & =\left\langle p(m, \cdot, 0), \tilde{\varphi}_{n}\right\rangle_{0} \\
& =\int_{-\infty}^{m} \mathrm{e}^{z^{2}} p(m, z, 0) \tilde{\varphi}_{n}(z) \mathrm{d} z \\
& =\frac{1}{\sqrt{\int_{-\infty}^{m} D_{\nu_{n, m}}^{2}(-y \sqrt{2}) \mathrm{d} y}} \int_{-\infty}^{m} \mathrm{e}^{\frac{z^{2}}{2}} p(m, z, 0) D_{\nu_{n, m}}(-z \sqrt{2}) \mathrm{d} z .
\end{aligned}
$$

It remains to determinate $c_{n}(t)$.

On one hand, $\frac{\partial p}{\partial t}(x, t)=\sum_{n=1}^{\infty} c_{n}^{\prime}(t) \tilde{\varphi}_{n}(x)$.

On the other hand, $\frac{\partial p}{\partial t}(x, t)=\mathbf{L}_{\mathrm{FP}} p(m, x, t)=\sum_{n=1}^{\infty} c_{n}(t) \mathbf{L}_{\mathrm{FP}} \tilde{\varphi}_{n}(x)=-\sum_{n=1}^{\infty} c_{n}(t) \nu_{n, m} \tilde{\varphi}_{n}(x)$.

By uniqueness of the decomposition, we have

$$
c_{n}^{\prime}(t)=-\nu_{n, m} c_{n}(t) .
$$

So

$$
c_{n}(t)=c_{n}(0) \mathrm{e}^{-\nu_{n, m} t} .
$$

Finally,

$$
\begin{gathered}
p(m, x, t)=\sum_{n=1}^{\infty}\left(\frac{1}{\sqrt{\int_{-\infty}^{m} D_{\nu_{n}, m}^{2}(-y \sqrt{2}) \mathrm{d} y}} \int_{-\infty}^{m} \mathrm{e}^{\frac{z^{2}}{2}} p(m, z, 0) D_{\nu_{n, m}}(-z \sqrt{2}) \mathrm{d} z\right) \\
\times \mathrm{e}^{-\nu_{n, m} t} \mathrm{e}^{-\frac{x^{2}}{2}} \frac{D_{\nu_{n, m}}(-x \sqrt{2})}{\sqrt{\int_{-\infty}^{m} D_{\nu_{n, m}}^{2}(-y \sqrt{2}) \mathrm{d} y}} \\
=\mathrm{e}^{-\frac{x^{2}}{2}} \sum_{n=1}^{\infty}\left(\int_{-\infty}^{m} \mathrm{e}^{\frac{z^{2}}{2}} p(m, z, 0) D_{\nu_{n, m}}(-z \sqrt{2}) \mathrm{d} z\right) \mathrm{e}^{-\nu_{n, m} t} \frac{D_{\nu_{n, m}}(-x \sqrt{2})}{\int_{-\infty}^{m} D_{\nu_{n, m}}^{2}(-y \sqrt{2}) \mathrm{d} y} .
\end{gathered}
$$

We can conclude with Proposition 6.3.

\section{Law of the supremum and its joint density with the process}

Natural extensions of this work is to check or extend the known results. First, we express the cumulative distribution function of the supremum. Then, we find PDE for the joint density of the process and its supremum.

\subsection{Cumulative distribution function of the supremum}

From Equation (3), it is easy to obtain the law of the supremum. The result is consistent with the law obtained using the link between the supremum of a process $X$ and the hitting time and the expression in [APP05].

Proposition 4.1 (Supremum law). For $m \in \mathbb{R}$ and $t \in \mathbb{R}_{+}^{*}$, the law of the supremum when $X_{0}=x_{0}$ is given by

$$
\mathbb{P}_{x_{0}}\left(\sup _{0 \leqslant s \leqslant t} X_{s}<m\right)=-\mathrm{e}^{\frac{k\left(x_{0}^{2}-m^{2}\right)}{2}} \sum_{n=1}^{\infty} \mathrm{e}^{-k \nu_{n, m} t} \frac{D_{\nu_{n, m}}\left(-x_{0} \sqrt{2 k}\right)}{\nu_{n, m} \partial_{\nu} D_{\nu_{n, m}}(-m \sqrt{2 k})},
$$

where $\left(\nu_{n, m}\right)_{n \in \mathbb{N}^{*}}$ is defined as in Theorem 2.1.

Proof : It is just integration and the application of Proposition 6.2.

Remark 4.2. Using the hitting time density named $p_{x_{0} \rightarrow m}^{(k)}$ in [APP05] (Eq. 31) and the link between hitting time and supremum, we can also find the expression (8). Indeed,

$$
\mathbb{P}_{x_{0}}\left(\sup _{s \leqslant t} X_{s}<m\right)=\mathbb{P}_{x_{0}}\left(T_{m}>t\right)=\int_{t}^{+\infty} p_{x_{0} \rightarrow m}^{(k)}(s) \mathrm{d} s
$$




\subsection{Results on the joint density}

With the expression of the joint density / distribution, we can find a system of PDEs satisfied by the joint density $\partial_{m} p$ of the process and its supremum. We take here $k=1$.

Proposition 4.3. In the deterministic case where $X_{0}=x_{0}$, the density $p$ satisfies the following PDEs :

$$
\forall m \in \mathbb{R}, \forall t \in \mathbb{R}_{+}^{*},\left\{\begin{array}{r}
-\partial_{t} \partial_{m} p(m, x, t)-\partial_{x}\left(x \partial_{m} p(m, x, t)\right)+\frac{1}{2} \partial_{x x}^{2} \partial_{m} p(m, x, t)=0, \quad x<m, \\
-m \partial_{m} p(m, m, t)-\frac{1}{2} \partial_{m}^{2} p(m, m, t)-\partial_{x} \partial_{m} p(m, m, t)=0, \\
-m \partial_{m} p(m, m, t)+\partial_{t} \partial_{m} \mathbb{P}_{x_{0}}\left(\sup _{0 \leqslant s \leqslant t} X_{s} \leqslant m\right)-\frac{1}{2} \partial_{x} \partial_{m} p(m, m, t)=0 .
\end{array}\right.
$$

Proof : For this proof, it is more convenient to use the Hermite function $H$ instead of the parabolic cylinder function $D$.

With the regularity of $H$ (see Appendix) and the regularity of $m \mapsto \nu_{n, m}$ (see Proposition 6.4), we can prove that the function $p$ is regular with respect to $x, m$ and $t$. Moreover, the order of the further partial derivatives does not matter.

Hence, from (1), we have the equation (9).

For the other equations, let us write the partial derivatives of $p$.

We denote

$$
h_{n}(m, t):=\frac{\mathrm{e}^{-\nu_{n, m} t} H_{\nu_{n, m}}\left(-x_{0}\right)}{\nu_{n, m} H_{\nu_{n, m}-1}(-m) \partial_{\nu} H_{\nu_{n, m}}(-m)} .
$$

Then, we have :

$$
p(m, x, t)=-\mathrm{e}^{m^{2}-x^{2}} \sum_{n=1}^{\infty} h_{n}(m, t) H_{\nu_{n, m}}(-x),
$$

and

$$
\mathbb{P}_{x_{0}}\left(\sup _{0 \leqslant s \leqslant t} X_{s} \leqslant m\right)=-\frac{1}{2} \sum_{n=1}^{\infty} \frac{h_{n}(m, t)}{\nu_{n, m}} \partial_{x} H_{\nu_{n, m}}(-m)
$$

We denote in the following $\nu_{n, m}^{\prime}=\partial_{m} \nu_{n, m}$ (see Proposition 6.4). We have

$$
\partial_{m} p(m, x, t)=-\mathrm{e}^{m^{2}-x^{2}}\left[\sum_{n=1}^{\infty} h_{n}(m, t)\left(2 m H_{\nu_{n, m}}(-x)+\partial_{m}\left(H_{\nu_{n, m}}(-x)\right)\right)+\sum_{n=1}^{\infty} \partial_{m} h_{n}(m, t) H_{\nu_{n, m}}(-x)\right] .
$$

Then, with Proposition 6.6, we have

$$
\partial_{m} p(m, m, t)=-\sum_{n=1}^{\infty} h_{n}(m, t) \partial_{x} H_{\nu_{n, m}}(-m)
$$

and

$$
\partial_{m} \partial_{x} p(m, m, t)=\sum_{n=1}^{\infty} h_{n}(m, t)\left(4 m \partial_{x} H_{\nu_{n, m}}(-m)+\nu_{n, m}^{\prime}\left(\partial_{\nu} \partial_{x} H\right)_{\nu_{n, m}}(-m)\right)+\sum_{n=1}^{\infty} \partial_{m} h_{n}(m, t) \partial_{x} H_{\nu_{n, m}}(-m)
$$

With (22), we have

$$
\begin{aligned}
\partial_{m} \partial_{t}\left(\mathbb{P}\left(\sup X_{s} \leqslant m\right)\right)= & \frac{1}{2} \sum_{n=1}^{\infty} h_{n}(m, t)\left[2 m \partial_{x} H_{\nu_{n, m}}(-m)+\nu_{n, m}^{\prime}\left(\partial_{\nu} \partial_{x} H\right)_{\nu_{n, m}}(-m)\right] \\
& +\frac{1}{2} \sum_{n=1}^{\infty} \partial_{m} h_{n}(m, t) \partial_{x} H_{\nu_{n, m}}(-m) .
\end{aligned}
$$

Equation (12) and Proposition 6.7 imply that

$$
\begin{aligned}
\partial_{m^{2}}^{2} p(m, m, t)= & \sum_{n=1}^{\infty} h_{n}(m, t)\left[-6 m \partial_{x} H_{\nu_{n, m}}(-m)-2 \nu_{n, m}^{\prime}\left(\partial_{\nu} \partial_{x} H\right)_{\nu_{n, m}}(-m)\right] \\
& -2 \sum_{n=1}^{\infty} \partial_{m} h_{n}(m, t) \partial_{x} H_{\nu_{n, m}}(-m) .
\end{aligned}
$$


Combining (13), (14) and (17), we obtain (10).

Combining (13), (15) and (14), we obtain (11).

Remark 4.4. It is Theorem 3.1 in [CP17]. This theorem is given for processes written as $X_{t}=A\left(X_{t}\right)+B_{t}$, where $A$ is a bounded function of class $\mathcal{C}^{1}$. Even if we are not under this hypothesis (for an OU process, $A(x)=-k x)$, the theorem is verified.

\section{Acknowledgements}

We thank Grégory Vial for his comments on this paper. 


\section{Appendix}

\subsection{Definitions and well-known properties on Hermite functions and Parabolic cylinder functions}

We recall here the definitions and some known results about parabolic cylinder functions and Hermite functions. These results can be found in chapters 4 and 10 of [LS72] and in Section 7 of [APP05].

Hermite functions For $\nu \in \mathbb{R}$, the Hermite function $H_{\nu}$ is defined, for all $x \in \mathbb{R}$ as

$$
H_{\nu}(x)=\frac{1}{2 \Gamma(-\nu)} \sum_{i=0}^{+\infty} \frac{(-1)^{i}}{i !} \Gamma\left(\frac{i-\nu}{2}\right)(2 x)^{i} .
$$

The Hermite function satisfies the following relations:

$$
\begin{gathered}
\partial_{x} H_{\nu}(x)=2 \nu H_{\nu-1}(x), \\
H_{\nu+1}(x)-2 x H_{\nu}(x)+2 \nu H_{\nu-1}(x)=0 .
\end{gathered}
$$

Combining (20) and (21), we have :

$$
\partial_{x^{2}}^{2} H_{\nu}(x)=-2 \nu H_{\nu}(x)+2 x \partial_{x} H_{\nu}(x) .
$$

When $\operatorname{Re}(\nu)>-1$,

$$
H_{\nu}(x)=\frac{2 \mathrm{e}^{x^{2}}}{\sqrt{\pi}} \int_{0}^{\infty} \mathrm{e}^{-t^{2}}(2 t)^{\nu} \cos \left(2 x t-\frac{\nu \pi}{2}\right) \mathrm{d} t .
$$

In particular, we have :

$$
H_{\nu}(0)=\frac{2^{\nu} \Gamma\left(\frac{1}{2}\right)}{\Gamma\left(\frac{1-\nu}{2}\right)}
$$

Then, for $n \in \mathbb{N}$,

$$
H_{2 n}(0)=(-1)^{n} \frac{(2 n) !}{n !}, \quad H_{2 n+1}(0)=0 .
$$

Remark 6.1 (Zeros of the function $\left.\nu \mapsto H_{\nu}(0)\right)$. The zeros $\left(\nu_{n, 0}\right)_{n}$ of the function $\nu \mapsto H_{\nu}(0)$ are known. They are the positive odd integers. Indeed, (24) indicates that $H_{\nu}(0) \neq 0$ when $\nu \notin \mathbb{N}$. Therefore, the zeros are among $\mathbb{N}$. Equation (25) allows to conclude.

Parabolic cylinder function The equation

$$
y^{\prime \prime}(x)+\left(-\frac{x^{2}}{4}+\frac{1}{2}+\nu\right) y(x)=0
$$

admits two real solutions. The parabolic cylinder function $D_{\nu}$ is defined as the solution of (26) with the property that $y(x) \underset{x \rightarrow+\infty}{\sim} x^{\nu} \mathrm{e}^{-x^{2} / 4}$. The other independent solution of $(26)$ is $x \mapsto D_{\nu}(-x)$.

We have the following relation

$$
\partial_{x} D_{\nu}(x)=\nu D_{\nu-1}(x)-\frac{x}{2} D_{\nu}(x)
$$

When $\operatorname{Re}(\nu)>-1$,

$$
D_{\nu}(x \sqrt{2})=\frac{2^{\frac{\nu}{2}+1} \mathrm{e}^{\frac{x^{2}}{2}}}{\sqrt{\pi}} \int_{0}^{\infty} \mathrm{e}^{-t^{2}} t^{\nu} \cos \left(2 x t-\frac{\nu \pi}{2}\right) \mathrm{d} t .
$$

We have, for $n \in \mathbb{N}$,

$$
\int_{-\infty}^{0} D_{n}^{2}(-x \sqrt{2}) \mathrm{d} x=n ! \frac{\sqrt{\pi}}{2}
$$

The parabolic cylinder function and the Hermite function are linked by the following relation :

$$
D_{\nu}(x \sqrt{2})=2^{-\frac{\nu}{2}} \mathrm{e}^{-\frac{x^{2}}{2}} H_{\nu}(x) .
$$




\subsection{Useful results}

In this part, we note $\nu_{\alpha}$ a zero of $\nu \mapsto D_{\nu}(-\alpha \sqrt{2})$. This is equivalent to a zero of $\nu \mapsto H_{\nu}(-\alpha)$. Thanks to (23), we have, for $x \in \mathbb{R}$ and when $\operatorname{Re}(\nu)>-1$,

$$
\begin{aligned}
\partial_{x} H_{\nu}(x) & =2 x H_{\nu}(x)-\frac{2 \mathrm{e}^{x^{2}}}{\sqrt{\pi}} \int_{0}^{\infty} \mathrm{e}^{-t^{2}}(2 t)^{\nu+1} \sin \left(2 x t-\frac{\nu \pi}{2}\right) \mathrm{d} t, \\
\partial_{\nu} H_{\nu}(x) & =\frac{2 \mathrm{e}^{x^{2}}}{\sqrt{\pi}} \int_{0}^{\infty} \mathrm{e}^{-t^{2}}(2 t)^{\nu}\left[\ln (2 t) \cos \left(2 x t-\frac{\nu \pi}{2}\right)+\frac{\pi}{2} \sin \left(2 x t-\frac{\nu \pi}{2}\right)\right] \mathrm{d} t, \\
\partial_{x} \partial_{\nu} H_{\nu}(x) & =2 x \partial_{\nu} H_{\nu}(x)-\frac{2 \mathrm{e}^{x^{2}}}{\sqrt{\pi}} \int_{0}^{\infty} \mathrm{e}^{-t^{2}}(2 t)^{\nu+1}\left[\ln (2 t) \sin \left(2 x t-\frac{\nu \pi}{2}\right)-\frac{\pi}{2} \cos \left(2 x t-\frac{\nu \pi}{2}\right)\right] \mathrm{d} t .
\end{aligned}
$$

Thanks to (26), we can prove by direct induction that $x \mapsto D_{\nu}(x)$ is a $\mathcal{C}^{\infty}(\mathbb{R})$ function. Then, so is $x \mapsto H_{\nu}(x)$. With the previous expressions, we can prove that all the derivatives with respect to $\nu$ exist and are continuous if $\nu \in \mathbb{R}_{+}$. Then, $\nu \mapsto H_{\nu}(x)$ is a $\mathcal{C}^{\infty}\left(\mathbb{R}_{+}\right)$function. The cross partial derivatives with respect to $\nu$ and $x$ also exist and are continuous. Then, we have the symmetry of second derivatives (Schwarz's theorem). We give here several results useful in the proofs.

Proposition 6.2. For all $\nu, \alpha \in \mathbb{R}$, we have :

$$
\int_{-\infty}^{\alpha} \mathrm{e}^{-\frac{x^{2}}{2}} D_{\nu}(-x \sqrt{2}) \mathrm{d} x=\frac{\mathrm{e}^{-\frac{\alpha^{2}}{2}}}{\sqrt{2}} D_{\nu-1}(-\alpha \sqrt{2}) .
$$

Proof : We prove the relation on $H$. Let us take $\nu, \alpha \in \mathbb{R}$. We have with (21) et (20):

$$
\begin{aligned}
\int_{-\infty}^{\alpha} \mathrm{e}^{-x^{2}} H_{\nu}(-x) \mathrm{d} x & =-\int_{-\infty}^{\alpha} \mathrm{e}^{-x^{2}}\left(2 x H_{\nu-1}(-x)+2(\nu-1) H_{\nu-2}(-x)\right) \mathrm{d} x \\
& =\int_{-\infty}^{\alpha} \partial_{x}\left(\mathrm{e}^{-x^{2}} H_{\nu-1}(-x)\right) \mathrm{d} x .
\end{aligned}
$$

Finally,

$$
\int_{-\infty}^{\alpha} \mathrm{e}^{-x^{2}} H_{\nu}(-x) \mathrm{d} x=\mathrm{e}^{-\alpha^{2}} H_{\nu-1}(-\alpha)
$$

Proposition 6.3. For all $\alpha \in \mathbb{R}$,

$$
\int_{-\infty}^{\alpha} D_{\nu_{\alpha}}^{2}(-x \sqrt{2}) \mathrm{d} x=-\frac{\nu_{\alpha}}{\sqrt{2}} D_{\nu_{\alpha}-1}(-\alpha \sqrt{2}) \partial_{\nu} D_{\nu_{\alpha}}(-\alpha \sqrt{2})
$$

Proof : We apply the method given in [Sle68].

Let set

$$
f: \mid \begin{array}{ccc}
\mathbb{R}^{2} & \longrightarrow & \mathbb{R} \\
(\nu, x) & \longmapsto & D_{\nu}(-x \sqrt{2})
\end{array} .
$$

Then, thanks to (26), the function $f$ is solution of the following equation, for all $\nu, x \in \mathbb{R}$ :

$$
\partial_{x^{2}}^{2} f(\nu, x)+\left(-x^{2}+1+2 \nu\right) f(\nu, x)=0 .
$$

We derive this equation with respect to $\nu$ and using the regularity of $H$ :

$$
\partial_{x^{2}}^{2} \partial_{\nu} f(\nu, x)+2 f(\nu, x)+\left(-x^{2}+1+2 \nu\right) \partial_{\nu} f(\nu, x)=0 .
$$

We multiply by $f$

$$
f(\nu, x) \partial_{x^{2}}^{2} \partial_{\nu} f(\nu, x)+2 f^{2}(\nu, x)+\left(-x^{2}+1+2 \nu\right) f(\nu, x) \partial_{\nu} f(\nu, x)=0 .
$$


We integrate with respect to $x$ :

$$
\begin{aligned}
\int_{-\infty}^{\alpha} f^{2}(\nu, x) \mathrm{d} x & =-\frac{1}{2}\left[\int_{-\infty}^{\alpha} f(\nu, x) \partial_{x^{2}}^{2} \partial_{\nu} f(\nu, x) \mathrm{d} x+\int_{-\infty}^{\alpha}\left(-x^{2}+1+2 \nu\right) f(\nu, x) \partial_{\nu} f(\nu, x) \mathrm{d} x\right] \\
& =-\frac{1}{2}\left[\left[f(\nu, x) \partial_{x} \partial_{\nu} f(\nu, x)\right]_{-\infty}^{\alpha}-\int_{-\infty}^{\alpha} \partial_{x} f(\nu, x) \partial_{x} \partial_{\nu} f(\nu, x) \mathrm{d} x-\int_{-\infty}^{\alpha} \partial_{x^{2}}^{2} f(\nu, x) \partial_{\nu} f(\nu, x) \mathrm{d} x\right] \\
& =-\frac{1}{2}\left[f(\nu, \alpha) \partial_{x} \partial_{\nu} f(\nu, \alpha)-\int_{-\infty}^{\alpha} \partial_{x}\left\{\partial_{x} f(\nu, x) \partial_{\nu} f(\nu, x)\right\} \mathrm{d} x\right] \\
& =-\frac{1}{2}\left[f(\nu, \alpha) \partial_{x} \partial_{\nu} f(\nu, \alpha)-\left[\partial_{x} f(\nu, x) \partial_{\nu} f(\nu, x)\right]_{-\infty}^{\alpha}\right] \\
& =-\frac{1}{2}\left[f(\nu, \alpha) \partial_{x} \partial_{\nu} f(\nu, \alpha)-\partial_{x} f(\nu, \alpha) \partial_{\nu} f(\nu, \alpha)\right] .
\end{aligned}
$$

Then, writing using the function $D$,

$$
\int_{-\infty}^{\alpha} D_{\nu_{\alpha}}^{2}(-x \sqrt{2}) \mathrm{d} x=\frac{-\sqrt{2}}{2} \partial_{x} D_{\nu_{\alpha}}(-\alpha \sqrt{2}) \partial_{\nu} D_{\nu_{\alpha}}(-\alpha \sqrt{2}) .
$$

The conclusion follows with (27).

Proposition 6.4. For all $\alpha \in \mathbb{R}$, there exists $g: \alpha \mapsto \nu_{\alpha}$, continuously differentiable on a neighbourhood of $\alpha$. We note $\nu_{\alpha}^{\prime}=g^{\prime}(\alpha)$. Then

$$
\nu_{\alpha}^{\prime}=\frac{\partial_{x} H_{\nu_{\alpha}}(-\alpha)}{\partial_{\nu} H_{\nu_{\alpha}}(-\alpha)}
$$

Proof : Let set

$$
f: \mid \begin{array}{ccc}
\mathbb{R}^{2} & \longrightarrow & \mathbb{R} \\
(\nu, x) & \longmapsto & D_{\nu}(-x \sqrt{2})
\end{array} .
$$

The function $f$ is a continuously differentiable function. We have $f\left(\nu_{\alpha}, \alpha\right)=0$. We also have $\partial_{\nu} f\left(\nu_{\alpha}, \alpha\right) \neq 0$ because of Proposition 6.3 and the fact that $\int_{-\infty}^{\alpha} D_{\nu}^{2}(-x \sqrt{2}) \mathrm{d} x \neq 0$.

Then, thanks to the implicit function theorem, there exists an open set $U$ of $\mathbb{R}$ containing $\alpha$ such that there exists an unique continuously differentiable function $g: U \mapsto \mathbb{R}$ such that $g(\alpha)=\nu_{\alpha}$ and for all $x \in U, f(g(x), x)=0$. Moreover, the partial derivative of $g$ in $U$ is given by

$$
g^{\prime}(x)=-\frac{\partial_{x} f(g(x), x)}{\partial_{\nu} f(g(x), x)} .
$$

Proposition 6.5. We have

$$
\begin{aligned}
\partial_{m}\left(H_{\nu_{m}}(x)\right)=\mathrm{e}^{x^{2}-m^{2}} \partial_{x} H_{\nu_{m}}(-m)-\frac{4 \nu_{m}^{\prime} \mathrm{e}^{x^{2}}}{\sqrt{\pi}} & \int_{0}^{\infty} \mathrm{e}^{-t^{2}}(2 t)^{\nu_{m}} \sin ((m+x) t) \times \\
& \quad\left[\ln (2 t) \sin \left((x-m) t-\frac{\nu_{m} \pi}{2}\right)-\frac{\pi}{2} \cos \left((x-m) t-\frac{\nu_{m} \pi}{2}\right)\right] \mathrm{d} t .
\end{aligned}
$$

Proof : Using Proposition 6.4 twice and (30) and (31), we have :

$$
\begin{aligned}
\partial_{m}\left(H_{\nu_{m}}(x)\right) & =\nu_{m}^{\prime} \partial_{\nu} H_{\nu_{m}}(x) \\
& =\nu_{m}^{\prime} \partial_{\nu} H_{\nu_{m}}(x)+\mathrm{e}^{x^{2}-m^{2}} \partial_{x} H_{\nu_{m}}(-m)-\mathrm{e}^{x^{2}-m^{2}} \partial_{x} H_{\nu_{m}}(-m) \\
& =\nu_{m}^{\prime} \partial_{\nu} H_{\nu_{m}}(x)+\mathrm{e}^{x^{2}-m^{2}} \partial_{x} H_{\nu_{m}}(-m)-\mathrm{e}^{x^{2}-m^{2}} \nu_{m}^{\prime}\left(\partial_{\nu} H\right)_{\nu_{m}}(-m) \\
& =\mathrm{e}^{x^{2}-m^{2}} \partial_{x} H_{\nu_{m}}(-m)-\frac{4 \nu_{m}^{\prime} \mathrm{e}^{x^{2}}}{\sqrt{\pi}} \int_{0}^{\infty} \mathrm{e}^{-t^{2}}(2 t)^{\nu_{m}} \sin ((m+x) t) \times \\
& \quad\left[\ln (2 t) \sin \left((x-m) t-\frac{\nu_{m} \pi}{2}\right)-\frac{\pi}{2} \cos \left((x-m) t-\frac{\nu_{m} \pi}{2}\right)\right] \mathrm{d} t .
\end{aligned}
$$

In particular, with $x=-m$, we have the following proposition : 
Proposition 6.6. For all $m \in \mathbb{R}$,

$$
\left.\partial_{m}\left(H_{\nu_{m}}(x)\right)\right|_{x=-m}=\partial_{x} H_{\nu_{m}}(-m) .
$$

We also have the following proposition :

Proposition 6.7. We have

$$
\left.\partial_{m^{2}}^{2}\left(H_{\nu_{m}}(x)\right)\right|_{x=-m}=2 \nu_{m}^{\prime} \partial_{\nu} \partial_{x} H_{\nu_{m}}(-m)+2 m \partial_{x} H_{\nu_{m}}(-m) .
$$

Proof : It is the same kind of calculus as in the proof of Proposition 6.5. We use the formula (32) and the fact that

$$
\begin{aligned}
\left.\partial_{m^{2}}^{2}\left(H_{\nu_{m}}(x)\right)\right|_{x=-m}=- & 2 m \partial_{x} H_{\nu_{m}}+\partial_{m}\left(\partial_{x} H_{\nu_{m}}(-m)\right) \\
& \quad-\frac{2 \nu_{m}^{\prime} \mathrm{e}^{m^{2}}}{\sqrt{\pi}} \int_{0}^{\infty} \mathrm{e}^{-t^{2}}(2 t)^{\nu_{m}+1}\left[\ln (2 t) \sin \left(-2 m t-\frac{\nu_{m} \pi}{2}\right)-\frac{\pi}{2} \cos \left(-2 m t-\frac{\nu_{m} \pi}{2}\right)\right] \mathrm{d} t .
\end{aligned}
$$

\section{References}

[APP05] L. Alili, P. Patie, and J. L. Pedersen. Representations of first hitting time density of an OrnsteinUhlenbeck process. Stochastic Models, 21(4):967-980, 2005.

[BD08] W. Boyce and R.C. DiPrima. Elementary Differential Equations and Boundary Value Problems. Wiley, 2008.

[Bré83] H. Brézis. Analyse fonctionelle. Collection Mathématiques appliquées pour la maîtrise. Masson, 1983.

[BS96] A.N. Borodin and P. Salminen. Handbook of Brownian motion: facts and formulae. Probability and its applications. Birkhäuser Verlag, 1996.

[CP17] L. Coutin and M. Pontier. PDE for joint law of the pair of a continuous diffusion and its running maximum. working paper hal-01591946, September 2017.

[CPN18] L. Coutin, M. Pontier, and W. Ngom. Joint distribution of a lévy process and its running supremum. J. Applied Probability, 55(2):488-512, 2018.

[DIRT13] F. Delarue, J. Inglis, S. Rubenthaler, and E. Tanré. First hitting times for general non-homogeneous 1d diffusion processes: density estimates in small time. working paper hal-00870991, October 2013.

[DK06] Ron A Doney and Andreas E Kyprianou. Overshoots and undershoots of lévy processes. The Annals of Applied Probability, pages 91-106, 2006.

[EH14] M. Escobar and J. Hernandez. A note on the distribution of multivariate brownian extrema. International Journal of Stochastic Analysis, 2014.

[Gar04] C. W. Gardiner. Handbook of stochastic methods for physics, chemistry and the natural sciences, volume 13 of Springer Series in Synergetics. Springer-Verlag, Berlin, third edition, 2004.

[HKR98] H. He, W. Keirstead, and J. Rebholz. Double lookbacks. Mathematical Finance, 8(3):201-228, 1998.

[JYC09] M. Jeanblanc, M. Yor, and M. Chesney. Mathematical Methods for Financial Markets. Springer Finance. Springer London, 2009.

[KS91] I. Karatzas and S. Shreve. Brownian Motion and Stochastic Calculus. Graduate Texts in Mathematics. Springer New York, 1991.

[LS72] N.N. Lebedev and R.A. Silverman. Special Functions and Their Applications. Dover Books on Mathematics. Dover Publications, 1972.

[Ngo16] Waly Ngom. Contributions to the study of default time of a Lévy process in complete observation and in incomplete Observation. Thesis tel-01466200, Université Paul Sabatier - Toulouse III, July 2016.

[RH89] H. Risken and H. Haken. The Fokker-Planck Equation: Methods of Solution and Applications Second Edition. Springer, 1989. 
[Sle68] BD Sleeman. On parabolic cylinder functions. IMA Journal of Applied Mathematics, 4(1):106-112, 1968.

[Som94] A. Sommerfeld. Sur la théorie analytique de la conduction thermique. Annales mathématiques, 45(2):263-277, 1894. 